\title{
Hiatal Surface Area's CT scan measurement is useful in hiatal hernia's treatment of bariatric patients
}

Cristian E. Boru, Marco Rengo, Angelo lossa, Francesco De Angelis, Matteo Massaro, Alessandra Spagnoli, Anna Guida, Andrea Laghi \& Gianfranco Silecchia

To cite this article: Cristian E. Boru, Marco Rengo, Angelo lossa, Francesco De Angelis, Matteo Massaro, Alessandra Spagnoli, Anna Guida, Andrea Laghi \& Gianfranco Silecchia (2019): Hiatal Surface Area's CT scan measurement is useful in hiatal hernia's treatment of bariatric patients, Minimally Invasive Therapy \& Allied Technologies, DOI: $10.1080 / 13645706.2019 .1683033$

To link to this article: https://doi.org/10.1080/13645706.2019.1683033

Published online: 31 Oct 2019.

Submit your article to this journal $\sqsubset$

Q View related articles $\longleftarrow$

View Crossmark data \lceil 


\title{
Hiatal Surface Area's CT scan measurement is useful in hiatal hernia's treatment of bariatric patients
}

\author{
Cristian E. Boru ${ }^{a}$ (D) Marco Rengo ${ }^{b}$ (D) Angelo lossa $^{a} \mathbb{D}^{D}$, Francesco De Angelis ${ }^{a}$, Matteo Massaro ${ }^{a}$, \\ Alessandra Spagnolic (D) Anna Guida ${ }^{\mathrm{a}}$ (D), Andrea Laghi ${ }^{\mathrm{b}}$ (D) and Gianfranco Silecchia ${ }^{\mathrm{a}}$



${ }^{\mathrm{b}}$ Department of Radiological Sciences, Oncology and Pathology, University La Sapienza of Rome, Latina, Italy; 'Department of Public Health and Infectious Diseases, University La Sapienza of Rome, Rome, Italy
\end{abstract}

ABSTRACT

Introduction: Hiatal surface area (HSA) measurement has been recently proposed as useful tool for tailored treatment of hiatal defects. Multidetector CT scan (MDCT) of the hiatal area was shown to be useful in hiatal hernia $(\mathrm{HH})$ management.

Purpose: MDCT preoperative HSA measurements validation as a useful method in the surgical repair decision making process of hiatal defects in candidates to antireflux \pm bariatric surgery.

Material and methods: Twenty-five obese patients (group A), candidates to laparoscopic cruroplasty \pm bariatric surgery, were prospectively evaluated preoperatively and after one year, using an original MDCT algorithm, compared with intraoperative HSA measurement. Twelve nonobese (group B) and 12 obese patients (group C), without GERD or $\mathrm{HH}$, were used as control groups.

Results: Median preoperative HSA was $7.9 \mathrm{~cm}^{2}$, (interquartile IQR 5.97-9.80) while intraoperative median HSA was $6 \mathrm{~cm}^{2}$ (6-9.5), $p=.84$. Postoperative median HSA was $3.8 \mathrm{~cm}^{2}(3.21-4.8)$, showing the efficacy of cruroplasty, comparable with HSA calculated in the control groups (3.98 for B and $3.69 \mathrm{~cm}^{2}$ for $C, p=.8547$ ). No statistically significant difference between MDCT preoperative measurement and intraoperative findings was observed.

Conclusions: Preliminary results demonstrate MDCT scan HSA measurements as a valid, noninvasive method to predict intraoperative findings. It allows the HSA monitoring in order to correlate the symptoms onset and failure of cruroplasty.

\section{ARTICLE HISTORY}

Received 12 April 2019

Accepted 17 September 2019

\section{KEYWORDS}

Hiatal surface area HSA; hiatal hernia; bariatric surgery; MDCT scan; posterior cruroplasty

\section{Introduction}

Measurement of the esophageal hiatal defect (hiatal surface area, HSA) has been proposed for the tailored surgical closure to prevent hiatal hernia recurrence and/or intrathoracic wrap migration in patients with symptomatic gastroesophageal reflux disease (GERD) referred to laparoscopic antireflux surgery (LARS) $[1,2]$. In 2007, Granderath highlighted the need for a standardized method of measurement of HSA. This was done intraoperatively by determining the twodimensional expanse of the hiatal orifice and performing cruroplasty (simple, reinforced, or tension-free) based on the obtained values through a mathematical formula [3].

Bariatric surgery has developed exponentially in the last 30 years, and reflux after different procedures (persistent or de novo) is becoming a major concern [4]. GERD is associated with obesity, and some of the surgical bariatric techniques might be "refluxogenic" per se; however, there is no worldwide consensus [5]. The guidelines of the international laparoscopic gastric sleeve (LSG) consensus conferences and the systematic bariatric surgery reviews recommend the repair of hiatal defects during bariatric procedures (without indication of a standard surgical technique) with the intent to reduce the incidence of postoperative symptomatic GERD [6,7].

Currently, the preoperative diagnosis of hiatal hernia $(\mathrm{HH})$ and GERD in an obese patient referred to bariatric surgery is based on the symptoms score, upper GI endoscopy (EGD) with multiple biopsies, and swallow radiological contrast studies. Data obtained with these methods should influence the surgical strategy, but there is no consensus and no obligation on the use of preoperative EGD and contrast studies [8]. On the other hand, there is a low

CONTACT Cristian E. Boru drcrisb@gmail.com Department of General Surgery and Bariatric Center of Excellence IFSO-EC, University La Sapienza of Rome, ICOT Hospital, Via F. Faggiana 1668, Latina, Italy

(C) 2019 Society of Medical Innovation and Technology 
correlation between the standard preoperative investigations such as barium swallow and the intraoperative findings for the HSA dimensions, the herniated stomach volume as well as the weakness of the pillars, even though demonstrated by a few studies [9]. Recently, the evaluation and measurement of the HSA using multidetector computer tomography (MDCT) scan with multiplanar reconstruction was proposed to determine the range of the normal HSA, HSA in obese patients, and the value of the HSA after surgical repair [10]. The MDCT scan was shown to be more accurate than conventional imaging in the evaluation of post LSG complications that may lead to revision surgery, including transhiatal sleeve migration [11].

Validation and standardization of this radiological method could have an impact on the management of obese patients referred to LARS, single or concomitant with bariatric surgery.

The aim of this prospective, monocentric pilot study was to demonstrate the efficacy and feasibility of the MDCT scan of HSA in the decision-making algorithm and follow-up of morbidly obese patients with GERD and HH who are candidates for laparoscopic bariatric surgery and/or LARS.

\section{Material and methods}

Twenty-five morbidly obese patients, candidates for laparoscopic posterior cruroplasty as a primary or revision procedure, single or concomitant with bariatric surgery for $\mathrm{HH}$ or GERD after bariatric surgery, were prospectively analyzed for measuring the HSA (group A). A radiological assessment of a preoperative MDCT was performed on a 64-row CT scanner (LightSpeed VCT, GE Medical Systems, Waukesha, WI, USA) as previously described [11]. The CT scans of the thorax, abdomen and pelvis were performed in the cranio-caudal direction after orally administering a solution of contrast media $(30 \mathrm{~mL}$ in $0.5 \mathrm{~L}$ water; sodium diatrizoate and meglumine diatrizoate solution, $370 \mathrm{mg} / \mathrm{mL}$; Gastrografin ${ }^{\circledR}$, Bracco) at rest and during the strain maneuver. Using a multiplanar reconstruction on the gastro-esophageal junction, one radiologist with over ten years of experience in abdominal imaging measured the HSA via a manually traced polygonal region of interest (ROI), anteroposterior and transverse diameters, and the thickness of the diaphragmatic pillar. The HSA included esophageal hiatus, both crus on each lateral edge. If the crural bundle separating the esophageal and aortic hiatuses was poorly visualized, then the anterior margin of the aorta was used to complete the circumference of the esophageal hiatus (Figure 1). This algorithm includes an original modification of the previously reported algorithm because the HSA was calculated during swallowing, allowing a more physiologic assessment of the HSA.

The scanner used in this study is equipped with iterative reconstruction algorithms. Such algorithms

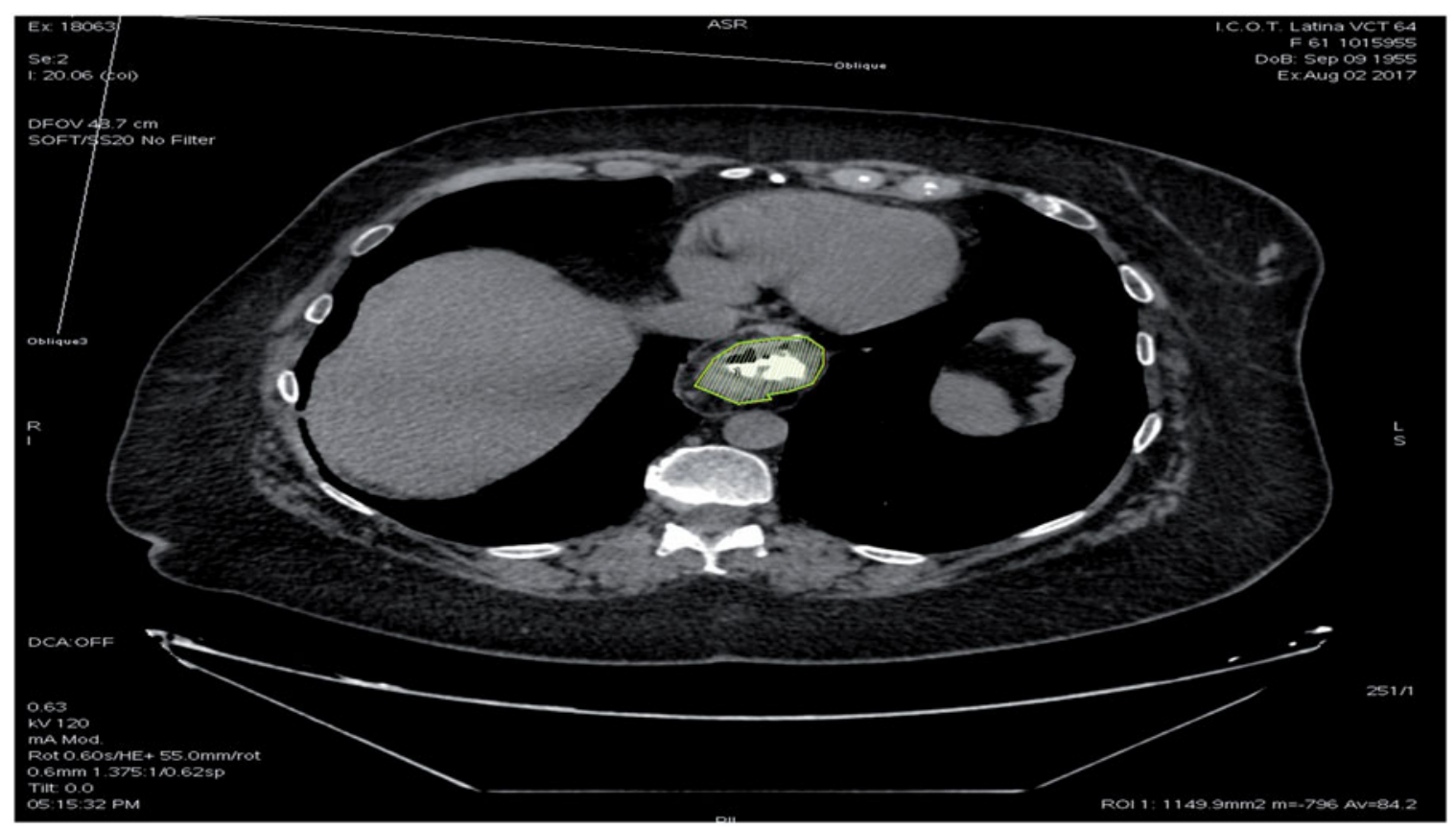

Figure 1. Hiatal surface area (HSA) measurement using MDCT scan, after reconstruction on three axes and tracking of the region of interest (ROI, shaded lines highlighted). Preoperative HSA: $11.7 \mathrm{~cm}^{2}$, in a patient with HH after LSG, with GERD and weight regain. Intraoperative measurement of HSA: $10 \mathrm{~cm}^{2}$. 


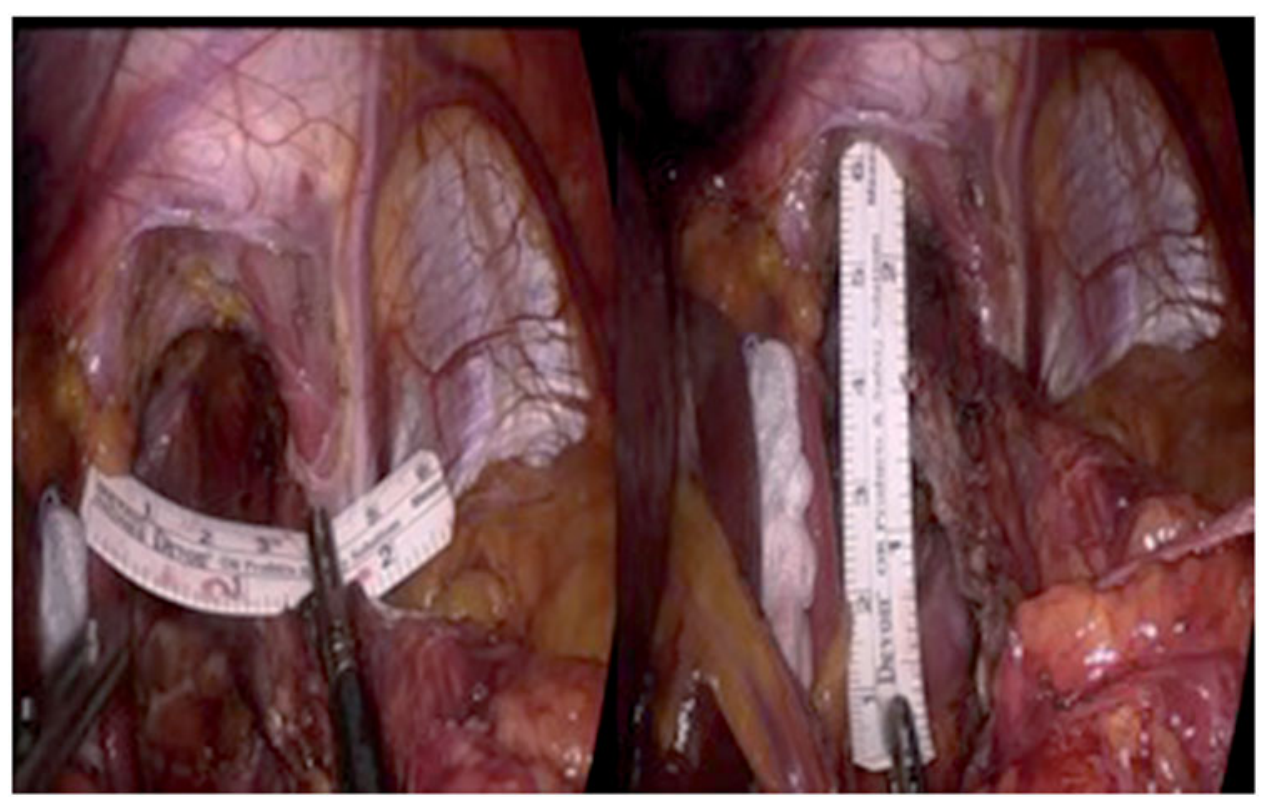

Figure 2. Intraoperative measurement of the hiatal defect, using two diameters: $d 1=$ the major horizontal distance between the two crura including their thickness and $d 2=$ the vertical distance between the pillars decussation and the superior edge of the esophageal hiatus. HSA was calculated using simplified rhomboid area formula: $\mathrm{HSA}=(\mathrm{d} 1 \times \mathrm{d} 2) / 2 \mathrm{in} \mathrm{cm}^{2}$.

allow reduction of the image noise when a low radiation dose acquisition protocol is used to obtain high image quality. In the study population, the average radiation exposure was $735.45( \pm 15.96) \mathrm{mGy} / \mathrm{cm}$, which can be considered extremely low, especially if we consider obese patients. In such patients, radiation exposure is higher than in normal-weight patients since a high radiation dose acquisition protocol is needed to ensure diagnostic image quality.

The study group included two patients with no previous surgical history and 23 patients previously subjected to different surgical procedures: LSG in 13 patients, LSG and cruroplasty in eight patients, and laparoscopic R-en-Y gastric bypass (LGBP) and cruroplasty in two patients. Symptomatic patients for GERD, who are candidates for different bariatric procedures with concomitant cruroplasty or revisional cruroplasty, were concomitantly studied with traditional methods, such as EGD, contrast studies, and ph-metry. During EGD, the HH was measured to evaluate the presumed differences between endoscopic and CT scan measurements. Esophagitis, neofundus formation, $\mathrm{HH}$, gastritis, and other lesions were registered.

After complete evaluation and preparation, patients were prepared for different surgical procedures. Evaluation of the HSA was completed during the laparoscopic surgery with the intraoperative measurement of the HSA, after the preparation of both the crura and the $\mathrm{HH}$ reduction when needed, with abdominalization of the esophagus for at least $3 \mathrm{~cm}$ and measurement of the major horizontal distance between the two crura, including their thickness (d1), and the vertical distance between the decussation pillars and the superior edge of the esophageal hiatus (d2). A simplified rhomboid area formula was used, as previously described: HSA $=(\mathrm{d} 1 \times \mathrm{d} 2) / 2$ in $\mathrm{cm}^{2}$ (Figure 2) [12].

We established a routine surgical protocol for the choice of the posterior cruroplasty technique (simple or reinforced). In case of intraoperative HSA measurement up to $4 \mathrm{~cm}^{2}$, we performed the simple posterior suture of the crura with two to three nonabsorbable stitches (Prolene ${ }^{\circledR}$ polypropylene 1/0, Ethicon Endosurgery, New Brunswick, NJ, USA), calibrated over a $48 \mathrm{Fr}$ bougie. In case of larger defects (HSA $>4 \mathrm{~cm}^{2}$ ), the posterior cruroplasty, performed with two to three non-absorbable stitches, always calibrated over a $48 \mathrm{Fr}$ bougie, is reinforced by the onlay positioning of a synthetic, bioabsorbable mesh, containing a copolymer of polyglycolic acid and trimethylene carbonate (Bio- $A^{\circledR}$, Gore ${ }^{\circledR}$, Flagstaff, AZ, USA), and fixed with absorbable suture and fibrin glue.

The Granderath's formula used for the intraoperative measurement of the HSA is a complex one, based on the calculation of the area of a circular sector (Figure 3) [1-3]. The correct formula for the area of a circular sector of radius $R$ and angle $\alpha_{0}$ (measured in radians) is

$$
A=\frac{R^{2} \alpha_{0}}{2}
$$




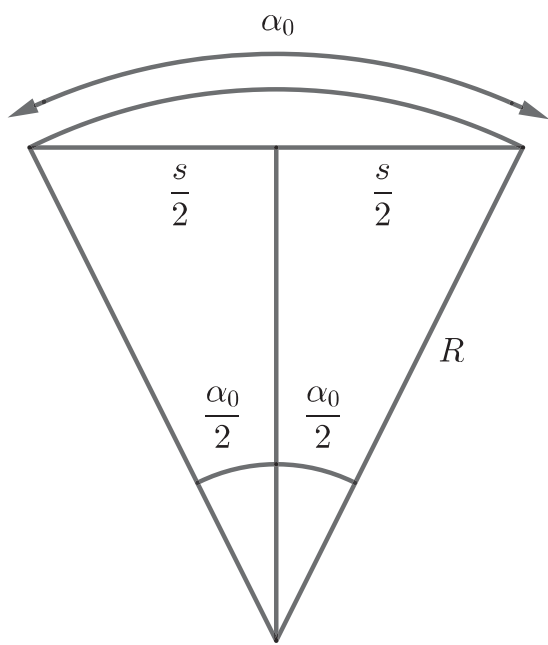

Figure 3. Calculation of the hiatal surface area (HSA) based on calculation of the area of a circular sector of radius $R$ and angle $\alpha_{0}$ (measured in radians). This calculation was proposed by Granderath as a model for intraoperative measurement of HSA (1-3).

If $s$ is the length of the chord, then

$$
\alpha_{0}=2 \arcsin \left(\frac{s}{2 R}\right)
$$

so that

$$
A=R^{2} \arcsin \left(\frac{s}{2 R}\right)
$$

Our approximating formula is

$$
A^{\prime}=\frac{R s}{2}
$$

Let us compare the two: writing $=\frac{s}{2 R}$, we have

$$
\frac{A^{\prime}}{A}=\frac{X}{\arcsin X}
$$

Assuming $\alpha_{0}$ to be less than a right angle (which is always the case, in practice), we have that $X$ may vary from 0 to $\frac{\sqrt{2}}{2}$, and hence,

$$
2 \frac{\sqrt{2}}{\pi} \leq \frac{X}{\arcsin X} \leq 1
$$

Since $2 \frac{\sqrt{2}}{\pi}=0.9003$, the error in the approximation is $<10 \%$. Therefore, even if our formula is a simplified one, it permits a more practical and rapid usage as the calculation error is not compromising the results.

Postoperative follow-up included clinical visits every three months in the first year and then twice a year. Phmetry and EGD were used in only three cases, where relapse or persistence of symptomatology was registered. The MDCT scan was performed 12 months postoperatively in all the patients using the same radiological protocol as described above.
In twelve non-obese subjects (group B) and twelve obese subjects (group C), without GERD, HH, or previous bariatric or hiatal surgery, measurement of the HSA was performed during MDCT scan prescribed for other medical reasons, and the radiological algorithm was adapted to their pathology. The values obtained were used to obtain the target score (HSA values in asymptomatic, normal-weight, and obese population), necessary for the statistical analysis.

\section{Statistical analysis}

Descriptive statistics were applied to summarize the variables. For continuous variables, median (interquartile range) and mean (standard deviation) were used. For discrete variables, the number of observations and frequency were reported. Friedman test was used to compare HSA measurement in-group A (preoperative CT scan, intraoperative measurement, and postoperative CT scan), followed by post-hoc Nemenyi-Test to adjust multiple comparisons. Kruskal-Wallis test was applied to compare the CT scan measurement of HSA between different groups (obese, non-obese, postoperative control), where $p<.05$ was considered statistically significant. The $\mathrm{R}$ statistical software version 3.5 .3 was used for all analyses.

\section{Results}

Twenty-five morbidly obese patients (mean BMI $42 \pm 6.2 \mathrm{~kg} / \mathrm{m}^{2}$, group A) and 24 control subjects with no $\mathrm{HH}$ or GERD were enrolled; mean BMI in group B (12 patients) was $24.17 \pm 2.79 \mathrm{~kg} / \mathrm{m}^{2}$ and $32.2 \pm 1.92 \mathrm{~kg} / \mathrm{m}^{2}$ in group C (12 patients). The demographic data are presented in Table 1. All surgical procedures were completed laparoscopically. The median HSA for preoperative HSA in group A was $7.9 \mathrm{~cm}^{2}$ (interquartile range IQR 5.97-9.8), while intraoperative HSA was $6 \mathrm{~cm}^{2}$ (IQR 6-9.5). Postoperative median HSA in group A (12 months after operation) was $3.8 \mathrm{~cm}^{2}$ (IQR 3.21-4.8). Median HSA in group B was $3.98 \mathrm{~cm}^{2}$ (IQR 3.32-5.05), while it was $3.69 \mathrm{~cm}^{2}$ (IQR 2.56-4.96) in group C. Median and mean values, together with IQR and standard deviations, are reported in Table 1.

No statistically significant difference was registered between preoperative radiological and intraoperative HSA measurements in group A $(p=.84)$; no statistically significant difference for mean HSA was found between group B and group C $(p=.8547)$. 
Table 1. Demographics and mean HSA measurements in study group and control patients.

\begin{tabular}{|c|c|c|c|c|c|c|}
\hline Patients & No & $\begin{array}{l}\text { Mean age } \\
\text { (years) }\end{array}$ & $\begin{array}{c}\text { Mean } \mathrm{BMI} \pm \mathrm{SD} \\
\left(\mathrm{kg} / \mathrm{m}^{2}\right)\end{array}$ & $\begin{array}{c}\text { Median HSA } \\
\text { preop }(+\mathrm{Qu}) \\
\text { Mean } \pm \mathrm{SD} \\
\left(\mathrm{cm}^{2}\right) \\
\end{array}$ & $\begin{array}{c}\text { Median HSA } \\
\text { intraop }(+\mathrm{Qu}) \\
\text { Mean } \pm \mathrm{SD} \\
\left(\mathrm{cm}^{2}\right)\end{array}$ & $\begin{array}{c}\text { Median HSA } \\
\text { postop }(+\mathrm{Qu}) \\
\text { Mean } \pm \mathrm{SD} \\
\left(\mathrm{cm}^{2}\right) \\
\end{array}$ \\
\hline Study group $\mathrm{A}$ obese, $\mathrm{HH} \pm \mathrm{GERD}$ & 25 & $50 \pm 12.1$ & $44.1 \pm 5.4$ & $\begin{array}{c}7.9 \\
(5.97-9.8) \\
8.25 \pm 2.91\end{array}$ & $\begin{array}{c}6 \\
(6-9.5) \\
7.68 \pm 2.58\end{array}$ & $\begin{array}{c}3.8 \\
(3.21-4.8) \\
4.1 \pm 1.48\end{array}$ \\
\hline $\begin{array}{l}\text { Control group B } \\
\text { Obesity, NO HH, NO surgery }\end{array}$ & 12 & $65 \pm 6.41$ & $32 \pm 1.92$ & $\begin{array}{c}3.98 \\
(3.32-5.05) \\
4.19 \pm 1.22\end{array}$ & - & - \\
\hline $\begin{array}{l}\text { Control group } \mathrm{C} \\
\quad \mathrm{NO} \text { Obesity, NO HH, NO surgery }\end{array}$ & 12 & $65.8 \pm 13.6$ & $24.17 \pm 2.79$ & $\begin{array}{c}3.69 \\
(2.56-4.96) \\
3.92 \pm 1.43\end{array}$ & - & - \\
\hline
\end{tabular}

Preop: MDCT scan preoperative measurement; intraop: intraoperative measurement; postop: 12 months MDCT scan postoperative measurement; IQR: interquartile range; SD: standard deviation.


Figure 4. (A) Box-plot showing HSA measurement preoperatively by MDCT scan ( $n=25$; median 7.9, range 5.97-9.8), intraoperatively manually $(n=25$; median 6 , range 6-9.5), and postoperatively by MDCT scan ( $n=25$; median 3.8, range 3.2-4.8). (B) Boxplot showing HSA measurement preoperatively by MDCT scan in group B non-obese patients $(n=12$; median 3.98, range 3.32-5.05) and in group $C$ obese patients ( $n=12$; median 3.69, range 2.56-4.96), and postoperatively by MDCT scan in study group A ( $n=25$; median 3.8, range 3.2-4.8). Filled dots represent mean values for each group.

Postoperative median HSA in group A was similar with groups $B$ and $C$, which had no $\mathrm{HH}$ or GERD patients $(p=.8547)$, due to $54 \%$ postoperative decrease in HSA.

Statistically significant differences were registered between group A (both preoperative and intraoperative measurements) and control groups $\mathrm{B}$ or $\mathrm{C}$ $(p=.4052)$. For group A, both preoperative and intraoperative measurements were carried out, before and after cruroplasty when the median HSA dropped from $7.9 \mathrm{~cm}^{2}$ to $3.8 \mathrm{~cm}^{2}$ and compared to postoperative CT scan control $(p<.0001)$. Statistics are presented in Figure 4.

The CT scan of HSA was used to calculate the dimensions of the two pillars of the diaphragm. In this study, mean values were $6 \pm 2.67 \mathrm{~mm}$ for the right crus and $7 \pm 2.06 \mathrm{~mm}$ for the left one. At the same time, gastric intrathoracic migration or $\mathrm{HH}$ was demonstrated in $96 \%$ of the patients from group A, with a mean ascendance of the esophagogastric junction of $5.5 \pm 0.63 \mathrm{~cm}$ that was raised to $7.4 \pm 1.78 \mathrm{~cm}$ after the requested straining maneuver.
Table 2. Preoperative upper Gl endoscopy (EGD) findings, with hiatal hernia measurement during the procedure.

\begin{tabular}{lc}
\hline & $\mathrm{N}^{\circ}$ Patients \\
\hline EGD Findings & \\
Esophagitis grade A & $2(8 \%)$ \\
Esophagitis grade B & $3(12 \%)$ \\
Neofundus + Midgastric stenosis & $1(4 \%)$ \\
Hypotonic cardias + mucosal hyperemia & $18(72 \%)$ \\
Hiatal Hernia & \\
$\leq 1,5 \mathrm{~cm}$ & $17(68 \%)$ \\
$>1,5 \mathrm{~cm} \leq 2,5 \mathrm{~cm}$ & $5(20 \%)$ \\
$>2,5 \mathrm{~cm}$ & $2(8 \%)$ \\
Intrathoracic migrated neofundus & $1(4 \%)$ \\
\hline
\end{tabular}

Data registered after preoperative EGD are shown in Table 2. One patient presented postoperative neofundus, migrated intrathoracic, and mediogastric stenosis; a small $\mathrm{HH}$ was diagnosed $(\leq 1.5 \mathrm{~cm})$ in 17 patients, including five patients with $\mathrm{HH}$ between 1.5 and $2.5 \mathrm{~cm}$, while two patients presented $\mathrm{HH}$ over $>2.5 \mathrm{~cm}$.

Surgical procedures included cruroplasty as standalone in seven cases or in concomitance with different 
bariatric procedures in 18 cases, as LSG or re-sleeve in three cases (12\%), as conversion to LGBP in 12 cases (48\%), and as conversion to laparoscopic minigastric bypass (LMGB) in three cases (12\%). Reinforced cruroplasty with bio-absorbable, synthetic mesh was performed in 12 patients (48\%) with enlarged HSA, as previously described $[12,13]$, where the mean HSA was $12.22 \pm 7.2 \mathrm{~cm}^{2}$ compared to the mean of all patients from group A $\left(7.68 \mathrm{~cm}^{2}\right.$, $p<.0001)$. In the surgical group two patients presented postoperative surgical complications: one pneumothorax treated with pleural drainage, with positive outcomes on the fourth postoperative day; one postoperative bleeding from the gastric suture line, initially treated conservatively and afterwards by laparoscopic hemostasis, with further regular evolution.

Clinical outcomes following surgical revision for GERD were quantified 12 months postoperatively in asymptomatic patients in 22 cases (88\%); mild symptoms with quality of life (Qol) unaffected and without the requirement of medication in two cases (8\%); and moderate symptoms with Qol unaffected and medication required in only one case (4\%). Outcomes of antireflux surgery are expressed both by the clinical outcomes and the $54 \%$ postoperative reduction in HSA, which remained stable 12 months postoperatively.

\section{Discussion}

Granderath first introduced the concept of measuring the HSA in 2007 by using an adapted intraoperative formula [3]. It was then modified by others, who introduced further intraoperative measuring methods, all with the goal to offer a tailored approach, based on the HSA values, for the antireflux surgery, which could be simple cruroplasty, reinforced with mesh or tension-free with mesh $[2,14,15]$. Our intraoperative formula, although simplified, provides relatively the same measurement as Granderath's formula, with a small error that does not alter the results, as has been mathematically demonstrated above.

The precedent intraoperative measurements described by different authors, obtained in vivo or after postmortem, are based on a surgical approach. This might represent a disadvantage due to incomplete preoperative informed consenst offered to the patient. This might lack important information about the existing pathology and the proposed surgical technique. A few studies have demonstrated that there is no real correlation between the preoperative data offered by traditional investigations and intraoperative evaluation [9]. Our intraoperative measurements were practically similar to the preoperative CT scan measurements $(p=.84)$.

Previously we showed that MDCT is an accurate noninvasive method for the identification of GERDrelated complications after bariatric surgery that might be missed by conventional radiography and endoscopy [11]. The use of MDCT has the advantage of high-spatial resolution and $3 \mathrm{D}$ features with a lower radiation dose exposure.

This is the first study that evaluates HSA in morbidly obese patients using CT scan, which has proved to be useful both as a minimally-invasive preoperative tool and as a method for the postoperative monitoring of surgical results. At the same time, this is the first study that demonstrates the utility of preoperative evaluation of HSA using CT scan, verified by the intraoperative measurement using a modified, simplified formula. Moreover, our study demonstrates a reduction in postoperative HSA values, similar to normal subjects or obese patients (without GERD or $\mathrm{HH}$ ).

Quyang presented a new method for the in vivo measurement of HSA using multiplanar, multislice MDCT to demonstrate a better correlation between $\mathrm{HH}$ and GERD, due to an enlarged HSA [10]. This was a retrospective study on 1,190 subjects that underlined a certain advantage of the procedure: the MDCT scan with $3 \mathrm{D}$ reconstruction was performed during a physiological status of the area (not during meals or strain maneuver), without the distension induced by the pneumoperitoneum, the relaxation induced by the anesthesia and the surgical dissection of the surrounding tissues during surgery. Subjects with $\mathrm{HH}$ had a larger mean HSA area than matched controls (6.9 vs. $2.5 \mathrm{~cm}^{2}, p<.0001$ ), while patients with GERD, without $\mathrm{HH}$, had a mean HSA of $3.0 \mathrm{~cm}^{2}$. In our study, the images are acquired in a cranio-caudal direction, immediately after the oral administration of a solution of iodinated contrast medium and tap water, ingested in no $>3 \mathrm{~min}$, and after requested strain maneuver, in order to evidence $\mathrm{HH}$ and intrathoracic migration. Therefore, this is a functional study, more appropriate for the evaluation of patients with suspected HH. In our experience, the value of HSA in normal and in obese subjects (mean values: 4.19 and $3.92 \mathrm{~cm}^{2}$, respectively) was higher than those reported by Quyang (mean $2.5 \mathrm{~cm}^{2}$ ), but similar to the dimensions found by other authors $[2,3,14]$ while mean HSA was definitely larger in patients with demonstrated $\mathrm{HH}$ (mean $8.3 \mathrm{~cm}^{2}$ ). As reported by Shamiyeh, a mean HSA of $5.84 \mathrm{~cm}^{2}$ was found in the cadavers of 
overweight (BMI $27 \mathrm{~kg} / \mathrm{m}^{2}$ ) subjects [14] with no $\mathrm{HH}$, but the difference might be explained by the physiological status of the tissues. Batirel proposed a postoperative value target of $2.5-3.0 \mathrm{~cm}^{2}$ after the surgical reparation of the hiatal defect [2].

One of the strengths of the study might be the measurement of the crura. The preoperative measurement might suggest that one or both the crus are weak and scarce, warranting the simplification of the decision-making process by proposing reinforcement of cruroplasty to the patient. The reinforced cruroplasty was performed in $48 \%$ of patients who presented with an enlarged HSA, and it was possible to preview this necessity preoperatively after informing the patients. Another aspect that should be mentioned is the intrathoracic migration of the stomach, demonstrated preoperatively in $96 \%$ of the patients by CT scan, and the values of migration seemed to be more important than those seen during EGDS when only eight patients presented real signs of $\mathrm{HH}(>1.5 \mathrm{~cm})$. The other 17 patients presented small, non-relevant endoscopic recurrence $(<1.5 \mathrm{~cm})$, with no clinical significance [16]. The added, preoperative information of the intrathoracic migration allows to inform the patients and adapt the surgical technique as well as to obtain imaging useful for the follow-up. Thereafter the migration was evaluated as part of the anatomic distortion of the HSA.

There are some limitations of our study. The study group is small and non-uniform. In addition, the study includes morbidly obese patients with or without previous crural repair and/or bariatric procedures that may alter the local anatomy. The handmade intraoperative measurement is not as precise as a machine or mathematic calculation, even if we mathematically demonstrated that the error is non-significant. In order to avoid unnecessary irradiation exposure, we used the data obtained from the radiological studies in obese and norm-weighted patients undergoing MDCT scan prescribed for other reasons. These subjects were considered as control sub-groups. Mean BMI in group C (obese patients) resulted inferior than in the study group, but it still represents a target population of our research.

In this pilot study, a complete preoperative evaluation (EGD, contrast studies, MDCT scan, GERD evaluation), as well as a postoperative follow-up (MDCT scan, GERD evaluation), was performed in all patients. Our intention is to expand this study to normal-weight patients who are candidates for laparoscopic antireflux surgery.

\section{Conclusions}

These preliminary results indicate that the measurement of the esophageal hiatus surface by MDCT scan could be a better radiological, non-invasive method for preoperative evaluation, due to the $3 \mathrm{D}$ high-resolution images and low-radiation dose. This would be helpful in deciding the need for antireflux and/or bariatric surgery, either primary or revisional, in the obese population. The initial results of this pilot study suggested the routine use of this method in the preoperative work-up, as well as for the postoperative HSA monitoring, in order to correlate the onset of symptoms and failure of cruroplasty. The results obtained are encouraging for a prolonged study and long-term follow-up in order to standardize the procedure.

\section{Acknowledgements}

We thank Prof. Alessandro Fonda, from University of Studies of Trieste, Italy, for assisting us with the mathematically demonstration of non-significant errors using the simplified formula during the intraoperative measurement of HSA.

The preliminary results of our study were presented as oral presentations at the National Congress of SICE (Italian Society of Endoscopic Surgery) in Milan, Italy 2017 and at the 22th World Congress of IFSO (International Federation for the Surgery of Obesity and Metabolic Disorders) in London, UK 2017.

The work described has not been published before; it is not under consideration for publication anywhere else. The publication has been approved by all co-authors, as well as by the responsible authorities at the institute where the work has been carried out. The publisher will not be held legally responsible should there be any claims for compensation.

\section{Ethical approval}

No ethical approval was required for this study. Permission was granted by the Institutional Review Board for this retrospective study. All procedures performed in studies involving human participants were in accordance with the ethical standards of the institutional research committee and with the 1964 Helsinki Declaration and its later amendments or comparable ethical standards. For this type of study, formal consent was not required. Informed consent was obtained from all individual participants included in the study.

This article does not contain any studies with animals performed by any of the authors.

\section{Author contributions}

All individual-participant data collected during this study will be available to access, after de-identification. Data and 
documents, including the study protocol, statistical analysis plan, technical details (like CT algorithm) and informed consent forms (in Italian) will be available. Data will only be available for use in individual participant data metaanalyses, and access will be provided to researchers after a proposal has been approved by an independent review committee identified for this purpose. Data will be available beginning at three months and ending at 24 months after publication of this article. Proposals should be directed to drcrisb@gmail.com; to gain access, data requesters will need to sign a data access agreement, and the de-identified database will be transferred by email.

\section{Declaration of interest}

All authors declare that they have no conflicts of interest. No funding was received for this study.

\section{ORCID}

Cristian E. Boru (D http://orcid.org/0000-0001-9171-4460 Marco Rengo (D) http://orcid.org/0000-0001-9087-5692 Angelo Iossa (D) http://orcid.org/0000-0002-6798-8100 Alessandra Spagnoli (iD http://orcid.org/0000-00027772-3130

Anna Guida (D) http://orcid.org/0000-0001-7792-3338

Andrea Laghi (D) http://orcid.org/0000-0002-3091-7819

Gianfranco Silecchia (D) http://orcid.org/0000-0003-

2356-0505

\section{References}

[1] Koch OO, Asche KU, Berger J, et al. Influence of the size of the hiatus on the rate of reherniation after laparoscopic fundoplication and refundopilication with mesh hiatoplasty. Surg Endosc. 2011;25(4): 1024-1030.

[2] Batirel HF, Uygur-Bayraicli O, Giral A, et al. The size of the esophageal hiatus in gastroesophageal reflux pathophysiology: outcome of intraoperative measurements. J Gastrointest Surg. 2010;14(1): $38-44$.

[3] Granderath F. Measurement of the esophageal hiatus by calculation of the hiatal surface area (HSA). Why, when and how? Surg Endosc. 2007;21(12): 2224-2225.

[4] Altieri MS, Pryor AD. Gastroesophageal reflux disease after bariatric procedures. Surg Clin North Am. 2015;95(3):579-591.

[5] Melissas J, Braghetto I, Molina JC, et al. Gastroesophageal reflux disease and sleeve gastrectomy. Obes Surg. 2015;25(12):2430-2435.
[6] Rosenthal RJ, International Sleeve Gastrectomy Expert Panel, Diaz AA, et al. International Sleeve Gastrectomy Expert Panel Consensus Statement: best practice guidelines based on experience of $>12,000$ cases. Surg Obes Relat Dis. 2012;8(1):8-19.

[7] Mahawar KK, Carr WR, Jennings $N$, et al. Simultaneous sleeve gastrectomy and hiatus hernia repair: a systematic review. Obes Surg. 2015;25(1): 159-166.

[8] Fried M, Yumuk V, Oppert JM, et al. European association for the study of obesity; international federation for the surgery of obesity - European chapter. Interdisciplinary European guidelines on metabolic and bariatric surgery. Obes Facts. 2013; 6(5):449-468.

[9] Koch OO, Schurich M, Antoniou SA, et al. Predictability of hiatal hernia/defect size: is there a correlation between pre- and intraoperative findings? Hernia. 2014;18(6):883-888.

[10] Ouyang W, Dass C, Zhao H, COPDGene Investigators, et al. Multiplanar MDCT measurement of esophageal hiatus surface area: association with hiatal hernia and GERD. Surg Endosc. 2016;30(6): 2465-2472.

[11] Rengo M, Bellini D, Iorio O, et al. Role of preoperative imaging with multidetector computed tomography in the management of patients with gastroesophageal reflux disease symptoms after laparoscopic sleeve gastrectomy. Obes Surg. 2013; 23(12):1981-1986.

[12] Ruscio S, Abdelgawad M, Badiali D, et al. Simple versus reinforced cruroplasty in patients submitted to concomitant laparoscopic sleeve gastrectomy: prospective evaluation in a bariatric center of excellence. Surg Endosc. 2016;30(6):2374-2381.

[13] Silecchia G, Iossa A, Cavallaro G, et al. Reinforcement of hiatal defect repair with absorbable mesh fixed with non-permanent devices. Minim Invasive Ther Allied Technol. 2014;23(5):302-308.

[14] Shamiyeh A, Szabo K, Granderath F, et al. The esophageal hiatus: what is the normal size? Surg Endosc. 2010;24(5):988-991.

[15] Hauters P, Bertrand C, Legrand M, et al. Traitement chirurgical du reflux gastro-oesophagien de l'adulte. EMC. Techniques chirurgicales - Appareil digestif. 2014;1-17.

[16] Oelschlager BK, Petersen RP, Brunt LM, et al. Laparoscopic paraesophageal hernia repair: defining long-term clinical and anatomic outcomes. J Gastrointest Surg. 2012;16(3):453-459. 